\title{
Rechtsprechung
}

\section{Zur Rechtsnatur des Spielzeitenvertrages und der Nichtverlängerung; Schiedsspruch Schweizerisches Bühnenschiedsgericht mit Sitz in Bern vom}

\section{März 2017}

- A. studierte Musikwissenschaften und arbeitete in der Folge als Dramaturgin und Regieassistentin. Im März 2013 stellte sie das Theater X. auf der Grundlage des geltenden Gesamtarbeitsvertrages (GAV) als „Dramaturgin Musiktheater \& Konzert" an. Vereinbart wurde insbesondere: „Der Arbeitsvertrag ist für die Spielzeiten 2013 / 2014 abgeschlossen. Das Arbeitsverhältnis beginnt am 1. Juli 2013 und endet am 30. Juni 2014. Für die Nichtverlängerung des Arbeitsvertrags wird auf Art. 29 Abs. 1 und 2 des Gesamtarbeitsvertrages verwiesen“. Im Dezember 2013 teilte das Theater X. der Dramaturgin mit, es erwäge den Arbeitsvertrag nicht über die Spielzeit 2013 / 2014 hinaus zu verlängern und lud sie unter Hinweis auf Art. 29 GAV zu einem Nichtverlängerungsgespräch ein. Nach dem Nichtverlängerungsgespräch teilte es A. im Januar 2014 schriftlich mit, dass ihr Arbeitsvertrag zum Ende der Spielzeit 2013/14 am 30. Juni 2014 auslaufe.

Mit Klage vom 8. Mai 2015 verlangte A. vor dem Schweizerischen Bühnenschiedsgericht die kostenfällige Feststellung, dass die Nichtverlängerungserklärung / Kündigung nichtig sei. Zudem sei die Beklagte zur Zahlung von CHF 20.426,70 brutto an sie zu verpflichten. Sie sei zum Zeitpunkt der Nichtverlängerungserklärung / Kündigung schwanger gewesen. Sie gehe von einem unbefristeten Arbeitsverhältnis aus, sodass Art. 336c Abs. 1 lit. c OR zur Anwendung gelange und das Arbeitsverhältnis über den 30. Juni 2014 hinaus bestehe. Ausgehend von der Geburt am 16. September 2014 habe die Kündigungssperrfrist 16 Wochen später, mithin am 6. Januar 2015, geendet. Damit das Arbeitsverhältnis nach der Spielzeit 2014 / 2015 hätte enden können, wäre eine Nichtverlängerungserklärung bis 31. Oktober 2014 nötig gewesen, was die laufende Sperrfrist verhindert habe. Damit könne das Arbeitsverhältnis frühestens nach dem Ende der Spielzeit 2015 / 2016, also am 30. Juni 2016, enden. Nach Abzug der Mutterschaftsentschädigung sowie der Gagen für zwei kurze Engagements stünden ihr bei Klageeinreichung CHF 20.426,70 brutto zu, die sie teilklageweise geltend mache. Von der Arbeitslosenkasse habe sie noch keine Leistungen erhalten.

Mit Klageantwort vom 10. Juni 2015 begehrte die Beklagte die kostenfällige Abweisung der Klage. Es liege ein sog. Spielzeitenvertrag vor. Die zwingende Befristung entspreche dem GAV Solo, sei Gewohnheitsrecht und sachgerecht. Als befristete Spielzeitenverträge endeten die Bühnenengagementsverträge nach Ablauf der vereinbarten Spielzeit, ohne dass hierzu eine Kündigung erforderlich wäre. Dass diejenige Partei, die den Bühnenengagementsvertrag in der darauffolgenden Spielzeit nicht weiterführen wolle, eine Nichtverlängerungserklärung ab- zugeben habe, sei ein weitverbreiteter und gewohnheitsrechtlich zu qualifizierender Brauch, der keine Kündigung, sondern lediglich eine Information bzw. bloße Mitteilung darstelle. Der Kündigungsschutz gem. Art. 336c OR finde nur auf die Kündigung unbefristeter Arbeitsverträge Anwendung. Damit habe das Arbeitsverhältnis am 30. Juni 2014 geendet.

„1.1. Gestützt auf Art. 50 (Schiedsklausel) des Gesamtarbeitsvertrages für das künstlerische Solopersonal vom 22. April 2014, in Kraft seit dem 1. Mai 2014 (nachfolgend GAV Solo 2014; Art. 2, Übergangs- und Schlussbestimmungen Abs. 1) haben der Schweizerische Bühnenverband (SBV) und der Schweizerische Bühnenkünstlerverband (SBKV) für die Regelung von Streitigkeiten im Bühnenbereich die Bühnenschiedsordnung (BSchO) vom 22. April 2014 (in Kraft seit 1. Mai 2014) erlassen und ein Bühnenschiedsgericht eingerichtet ( $\$ 1 \mathrm{BSchO}$ ). Die BSchO „regelt alle Streitigkeiten, die sich aus dem Arbeitsverhältnis zwischen Bühnenleitung und künstlerischem Personal ergeben, das dem GAV untersteht" (§ 2 Abs. 1 BSchO). „Die Zuständigkeit des Schiedsgerichts kann auch vereinbart werden für Streitigkeiten, die sich aus dem Arbeitsverhältnis zwischen Bühnenleitung und anderem Personal als den Bühnenmitgliedern ergeben" ( $§$ 2 Abs. 3 BSchO). Bezüglich des anwendbaren Verfahrensrechts bestimmt $\S 7$ BSchO, dass sich das Verfahren in hierarchischer Reihenfolge nach den zwingenden Bestimmungen des 12. Kapitels des IPRG (internationale Schiedsgerichtsbarkeit), nach den Bestimmungen der BSchO sowie nach den verfahrensleitenden Anordnungen des Schiedsgerichts, die dieses in der Regel nach Rücksprache mit den Parteien verabschiedet, richtet.

1.2. Die Parteien schlossen am 8. März 2013 einen Arbeitsvertrag „auf der Grundlage des zwischen dem Schweizerischen Bühnenverband und dem Schweizerischen Bühnenkünstlerverband geltenden Gesamtarbeitsvertrages (GAV)" ab [...]. Zu jenem Zeitpunkt war der GAV für das künstlerische Solopersonal 1981 in der Fassung von 1997 (GAV Solo 1981/1997) in Kraft. Der GAV Solo 2014 hat mit Inkrafttreten am 1. Mai 2014 den GAV Solo 1981 / 1997 ersetzt und ist für alle damals schon bestehenden Spielzeitenverträge verbindlich (Übergangs- und Schlussbestimmungen Abs. 1 und 2). Unstreitig ist zwischen den Parteien, dass ihr Arbeitsverhältnis am 1. Mai 2014 noch nicht beendet war, sodass der GAV Solo 2014 darauf anwendbar ist.

1.3. Gemäss Arbeitsvertrag wurde die Klägerin als Dramaturgin Musiktheater \& Konzert angestellt und als Bühnenmitglied bezeichnet (Arbeitsvertrag Ingress, Ziff. I. und III.). Zudem anerkannten „beide Vertragsparteien für den Fall allfälliger Strei- 
tigkeiten aus dem Arbeitsverhältnis die von beiden Verbänden vereinbarte Bühnenschiedsordnung und [verzichteten] auf den ordentlichen Rechtsweg" (Arbeitsvertrag Ziff. V.)

[...]. Nach Art. 1 Abs. 1 lit. b und c GAV wird das künstlerische Solopersonal als Bühnenmitglieder bezeichnet. Zu untersuchen ist, ob die Klägerin als Bühnenmitglied gelten kann.

1.4. Nach Art. 1 Abs. 3 GAV sind Laien, Statisten, Assistenten, Hospitanten, Lernende, Studierende, Schüler, Praktikanten keine Bühnenmitglieder. Sie alle gelten bloss als Mitwirkende in Bühnenaufführungen, auf die der GAV keine Anwendung findet (Art. 1 Abs. 3 GAV). Bühnenmitglieder sind daher in erster Linie darstellende Bühnenkünstler, insbesondere Schauspieler, Tänzer oder Sänger. Daneben werden an Theatern nicht darstellende Bühnenkünstler beschäftigt, die ebenfalls Bühnenmitglieder sind. Entscheidend ist, dass diese Personen „durch ihre künstlerische Tätigkeit unmittelbar an der Erarbeitung und konzeptionellen Umsetzung eines Werkes mitarbeiten“ (Bernhard Opolony Die Befristung von Bühnenarbeitsverhältnissen, in Zeitschrift für Arbeitsrecht, ZfA, 2000,179 ff., 181 mit Hinweisen auf die Rechtsprechung). Dazu gehören insbesondere auch Dramaturgen (Peter Mosimann Bühnenarbeitsrecht, in: Mosimann/ Renold/Rascher (Hrsg.) Kultur, Kunst, Recht, Schweizerisches und internationales Recht, 2009, S. 672; § 1 Abs. 2 des deutschen Normalvertrages Bühne; Christoph Nix/Jan Hegemann/Rolf C. Hemke (Hrsg.) Normalvertrag Bühne, Handkommentar, 2008, § 1 Rn. 3, S. 31, und § 53 Rn. 7, S. 206; Paul Dienstag/Alexander Elster Handbuch des Deutschen Theater-, Film-, Musik- und Artistenrechts, 1932, S. 475). Als solche sind sie an der Spielplangestaltung der Theater beteiligt, suchen und lesen geeignete Bühnenstücke, die sie in literarischer und musikalischer Hinsicht für die Regie bearbeiten (http://www.theaterschweiz.ch/de/sbv/ theaterberufe/berufsportraets/dramaturgin/; Dienstag/Elster S. 476). Auch gemäss GAV Solo 2014 sind sie daher zu den Bühnenkünstlern und Bühnenmitgliedern zu zählen. Auf Rechtsstreitigkeiten zwischen ihnen und der Bühnenleitung findet demzufolge ohne Weiteres die BSchO Anwendung, die die sachliche Zuständigkeit des Bühnenschiedsgerichts zur Beurteilung der vorliegenden Streitigkeit der Parteien begründet.

2.1. Strittig unter den Parteien ist die Qualifikation ihres Arbeitsverhältnisses. Die Klägerin geht von einem unbefristeten Arbeitsvertrag aus, auf den der zeitliche Kündigungsschutz nach Art. 336c Obligationenrecht (OR, SR 220) Anwendung finde. Die Beklagte andererseits stellt darauf ab, dass ein Spielzeitenvertrag und damit ein befristeter Arbeitsvertrag vorliege.

2.2. Beide Parteien haben Wohnsitz bzw. Sitz in der Schweiz. Der Arbeitsort befand sich ebenfalls in der Schweiz. Damit liegt ein Binnenarbeitsverhältnis vor, auf das schweizerisches Recht zur Anwendung gelangt. Nach $\S 6$ Abs. 1 BSchO entscheidet das Bühnenschiedsgericht in hierarchischer Reihenfolge nach den Bestimmungen des GAV, den einschlägigen Bühnenusanzen und den Bestimmungen des Schweizerischen Rechts. Eine Rechtswahl ist ausgeschlossen ( $§ 6$ Abs. 2 BSchO).
Die Art. 334 ff. OR unterscheiden zwischen befristeten und unbefristeten Arbeitsverhältnissen. Nach Art. 334 Abs. 1 OR endigt ein befristetes Arbeitsverhältnis ohne Kündigung, während nach den Art. 335 ff. OR unbefristete Arbeitsverhältnisse zu kündigen sind, um sie zu beendigen. Falls ein befristetes Arbeitsverhältnis nach Ablauf der vereinbarten Dauer stillschweigend fortgesetzt wird, ergeben sich die gesetzlichen Vermutungen, dass es nicht neu begründet, sondern verlängert und zudem in ein unbefristetes umgewandelt wird (Art. 334 Abs. 2 OR; Wolfgang Portmann/Roger Rudolph Basler Kommentar, OR I, 6. Aufl. 2015, Art. 334 OR Rn. 12 f., S. 2045). Dies hat zur Folge, dass es danach zu kündigen ist, um es zu beendigen. Die beiden gesetzlichen Vermutungen können allerdings widerlegt werden (BGE C 293/00 E. 2a; BSK-Portmann/Rudolph aaO).

2.3. Vorliegend ist von beiden Parteien anerkannt, dass der von ihnen abgeschlossene schriftliche Arbeitsvertrag bestimmt, dass dieser "für die Spielzeiten 2013/2014 abgeschlossen [ist]" und dass „das Arbeitsverhältnis [...] am 1. Juli 2013 [beginnt] und [...] am 30. Juni 2014 [endet]". Zudem verweist der Arbeitsvertrag für die Nichtverlängerung auf Art. 29 Abs. 1 und 2 GAV [...]. Die Klägerin erblickt darin eine Minimalfrist, weil das Arbeitsverhältnis nur dann nicht stillschweigend verlängert werde, wenn eine Partei vorher eine Nichtverlängerungserklärung abgebe. Dadurch werde das Arbeitsverhältnis zu einem unbefristeten [...]. Die Spielzeitenverträge seien damit jeweils auf das Ende einer Spielzeit kündbar [...]. Die Beklagte widerspricht dieser Sichtweise, weil die Nichtverlängerungserklärung keine Kündigung, sondern bloss eine Obliegenheit sei, ohne deren Abgabe das Arbeitsverhältnis bloss um eine weitere Spielzeit verlängert werde [...]. Zu untersuchen ist daher zunächst, ob das Arbeitsverhältnis befristet oder unbefristet ist.

2.4. Die Befristung eines Arbeitsvertrages kann ausdrücklich oder stillschweigend vereinbart werden, wobei der Wille der Vertragsparteien zur Befristung bestimmt und unzweideutig zum Ausdruck kommen muss (GSGer BS, BJM 2001, S. 60). Anderenfalls liegt ein unbefristetes Arbeitsverhältnis vor (BGE 4A_270/2014 E. 4.4; 4A_531/2008 E. 2.1, 4C.62/2001 E. 1C). Die Befristung kann sich aus der Dauer, dem Endtermin oder dem Zweck der Anstellung oder durch den Eintritt eines künftigen Ereignisses ergeben, von dem der Zeitpunkt des Eintritts ungewiss ist (BGer. 4C.62/2001 E. 2b; OGer LU, JAR 2000, S. 365 E. 4.5). Sie muss objektiv bestimmt oder bestimmbar sein. Ihr Eintritt darf nicht vom Willen einer Vertragspartei abhängen (BGer. 4A_270/2014 E. 4.4; 4C.62/2001 E. 2b). Das Vertragsende muss für Arbeitgeber und Arbeitnehmer ungefähr voraussehbar sein, um den zeitlichen Umfang ihrer Bindung abschätzen (OGer LU, JAR 2000, S. 365 E. 4.5) und rechtzeitig die erforderlichen Massnahmen für die Zeit danach vorkehren zu können (BGer. 4C.22/2000 E. 2b = Pra 2001 Nr. 31). Ein Teil der Lehre verlangt zudem, dass die Befristung sachlich gerechtfertigt ist (Alexander J. P. Ertl Das befristete Arbeitsverhältnis in Theorie \& Praxis, 2015, S. 16 Rn. 65.; BK-Rehbinder/Stöckli Der Arbeitsvertrag Art. 331-355 und Art. 361-362 OR, 2. Aufl. 2014, Art. 334 Rn. 12, S. 138; Thomas Brender Rechtsprobleme des befristeten Arbeitsvertra- 
ges, 1976, S. 113 ff.; ablehnend: ZK-Staehelin Der Arbeitsvertrag: Art. 330b-355 OR, Art. 361-362 OR, 4. Aufl. 2014, Art. 334 Rn. 4, S. 75; Jürg Brühwiler Einzelarbeitsvertrag, 2014, Art. 334 OR Rn. 6, S. 371; vgl. BSK-Portmann/Rudolph Art. 334 OR Rn. 8, S. 2043; Geiser/Müller Arbeitsrecht in der Schweiz, 3. Aufl. 2015, S. 225 ff. Rn. 541 ff.; Streiff/von Kaenel/Rudolph Arbeitsvertrag, Praxiskommentar zu Art. 319-362 OR, 7. Aufl. 2012, Art. 334 N 1 ff., S. $880 \mathrm{ff}$.). Ein unzulässig befristetes Arbeitsverhältnis wird als ein unbefristetes behandelt (BGE JAR 2000, S. 105 E. 2c).

Es ist anerkannt, dass befristete Arbeitsverhältnisse aufgrund einer Vereinbarung der Arbeitsvertragsparteien befristet fortgesetzt bzw. verlängert werden können. Erlaubt ist den Parteien auch der Abschluss eines weiteren befristeten Arbeitsverhältnisses, das sich unmittelbar an das zu Ende gehende Arbeitsverhältnis anschliesst (BGE 139 III 145 E. 4.1 = Pra 2013 Nr. 96; Daniel Durante Le renouvellement des contrats de durée, 2016, S. 60 Rn. 265). Letzteres führt zu einem Kettenarbeitsverhältnis, das zulässig ist, wenn dafür sachliche Gründe vorliegen. Hingegen darf damit nicht der Zweck verfolgt werden, die Anwendung der Kündigungsschutzbestimmungen (Art. 335a ff. OR) zu umgehen oder die Entstehung von Ansprüchen des Arbeitnehmers, die von einer Minimaldauer des Arbeitsverhältnisses abhängig sind (z.B. Art. 324a OR), zu verhindern, was rechtsmissbräuchlich wäre (Art. 2 Abs. 2 ZGB; BGE 139 III 145 E. $4.1=$ Pra 2013 Nr. 96; 129 III 618 E. 6.2 = Pra 2004 Nr. 66; 119 V 46 E. 1C = Pra 1993 Nr. 241) und dazu führte, dass das Kettenarbeitsverhältnis als ein einheitliches, unbefristetes Arbeitsverhältnis behandelt würde (Rémy Wyler/Virginie Panchaud La qualification du travail de l'artiste et ses conséquences, in: Bettina Kahil-Wolff/ Stéphanie Perrenoud (Hrsg.) Les acteurs culturels en droit social, Bern 2012, S. 51 ff., 68 f.; Durante, S. 61 Rn. 268). Das Bundesgericht geht davon aus, dass im Allgemeinen das Aneinanderreihen zweier befristeter Verträge noch keinen Rechtsmissbrauch darstellt, obwohl die Anzahl der Verträge für sich allein nicht bestimmend ist (BGE 139 III 145 E. 4.1 = Pra 2013 Nr. 96; BGer. 4C.51/1999 E. 2; vgl. Wyler/Panchaud S. 69: Hypothese des Vorliegens eines Rechtsmissbrauchs bei mehr als zwei Verträgen). Eine allfällige Gesetzesumgehung hat diejenige Partei zu beweisen, die sie behauptet (BGE 139 III 145 E. 4.2.4 = Pra 2013 Nr. 96). „Sachliche Gründe für eine Aneinanderreihung mehrerer befristeter Arbeitsverträge bejahen Lehre und Rechtsprechung insbesondere bei der Anstellung von Künstlern (Schauspielern, Musikern, Sängern etc.) [...]. Als sachliche Motive anzuerkennen sind grundsätzlich Besonderheiten des Arbeitsverhältnisses oder besondere betriebliche Umstände wie beim Bühnenengagement [...]" (BGer. 2P.26/2007 E. 3.7.).

2.5.1.Der Arbeitsvertrag der Parteien erscheint vom seinem Wortlaut her durch den Bezug auf eine einzelne Spielzeit und durch die datumsmässig klar bestimmte Dauer als befristeter Vertrag angelegt zu sein. Die Vertragsauslegung erfolgt jedoch aufgrund des übereinstimmenden wirklichen Willens der Parteien (Art. 18 Abs. 1 OR) und, falls ein solcher nicht festgestellt werden kann, nach dem Vertrauensprinzip (Art. 2 Abs. 1 ZGB). Dabei ist der Erklärungswortlaut nicht isoliert, sondern aus dem konkreten Sinngefüge heraus zu beurteilen. Maßgebend ist der von der erklärenden Partei verfolgte Regelungszweck, wie ihn der Erklärungsempfänger nach Treu und Glauben (Art. 2 Abs. 1 ZGB) verstehen durfte und musste (BGE 140 III 391 E. 2.3).

Der Arbeitsvertrag der Parteien steht allerdings nicht alleine da. Er wurde „auf der Grundlage des zwischen dem Schweizerischen Bühnenverband und dem Schweizerischen Bühnenkünstlerverband geltenden Gesamtarbeitsvertrages (GAV)“ abgeschlossen [...]. Art. 1 des GAV Solo 2014 bestimmt, dass der GAV "die Rechtsbeziehungen zwischen den dem SBV angeschlossenen Theatern (Bühnenleitungen) und dem von ihnen im Spielzeitenvertrag beschäftigten künstlerischen Solopersonal (Bühnenmitgliedern)" ordnet (Abs. 1 lit. b). Da die beklagte Partei dem SBV angeschlossen ist, kommt der GAV Solo 2014 als gesamtarbeitsvertragliche und nicht als einzelarbeitsvertragliche Regelung auf das Arbeitsverhältnis zur Anwendung.

2.5.2. Normative Bestimmungen eines GAV regeln als autonomes Satzungsrecht Rechte und Pflichten der dem GAV unterworfenen Arbeitnehmer und Arbeitgeber. Sie wirken unmittelbar und sind, soweit der GAV nichts anderes bestimmt (Art. 357 Abs. 1 OR), unabdingbar. Entsprechendes bestimmt Art. 3 Abs. 1 des vorliegend anwendbaren GAV. Gemäss Günstigkeitsprinzip können jedoch zugunsten der Arbeitnehmer im Verhältnis zum GAV günstigere Regelungen getroffen werden (Art. 357 Abs. 2 OR), was auch Art. 3 Abs. 2 GAV Solo 2014 ausdrücklich festlegt. Gesamtarbeitsverträge dürfen ferner nicht gegen zwingendes materielles Gesetzesrecht verstossen. Anderenfalls sind sie unwirksam. Nach Art. 358 OR gilt auch im Verhältnis zwischen GAV und Gesetz das Günstigkeitsprinzip (BK-Stöckli Art. 356 OR N. 118, S. 121). Der vorliegend interessierende Art. 334 OR ist weder absolut noch relativ zwingender Natur (Art. 361 f. OR).

2.5.3. Bezüglich der Frage, ob die Arbeitsverträge nach dem GAV Solo 2014 befristet oder unbefristet sind, finden sich darin verschiedene Vorschriften. Nach Art. 7 GAV Solo 2014 muss im Arbeitsvertrag die Dauer des Arbeitsverhältnisses vereinbart werden (lit. c). Art. 8 Abs. 2 GAV Solo 2014 erklärt Vertragsbestimmungen für nichtig, mit der sich die Bühnenleitung das Recht vorbehält, durch einseitige Erklärung den Vertrag über die vereinbarte Zeit hinaus zu verlängern. Art. 9 Abs. 1 GAV Solo 2014 schreibt ferner vor, dass für den Abschluss von Arbeitsverträgen das von den vertragsschliessenden Verbänden verfasste Arbeitsvertragsformular zu verwenden ist. Dieses dem GAV Solo 2014 angefügte Formular (GAV Solo 2014, S. 46 f.) bezeichnet den Solistenvertrag in Klammern als "Spielzeitenvertrag". Unter Ziff. II ist die massgebende Spielzeit, für die der betreffende Arbeitsvertrag abgeschlossen wird, sowie anzugeben, wann das Arbeitsverhältnis beginnt. Ausserdem verweist Ziff. Il des Formularvertrages „für die Nichtverlängerung des Vertrages" ausdrücklich auf Art. 29 GAV Solo 2014. Diese Vorschrift bestimmt, dass eine Vertragspartei, die den Arbeitsvertrag „für die nächste Spielzeit nicht zu erneuern gedenkt", dies der anderen Vertragspartei im ersten Vertragsjahr bis spätestens 31. Januar schriftlich zu erklären hat. Ohne eine solche fristgerechte 
schriftliche Erklärung gelte der bestehende Vertrag als für die nächste Spielzeit erneuert (Abs. 1). „Bevor die Bühnenleitung die Nichtverlängerung des Vertrages rechtswirksam erklären kann, hat sie das Bühnenmitglied mindestens 14 Tage vor dem Nichtverlängerungstermin schriftlich zu einem Informationsgespräch über die Nichtverlängerungsabsicht einzuladen“" (Art. 29 Abs. 2 GAV).

2.5.4.Es zeigt sich aufgrund der vorstehenden Ziff. 2.3. und 2.5.3., dass der vorliegende Arbeitsvertrag hinsichtlich der Frage, wie die Vertragsdauer bestimmt wird, sich an den GAV Solo 2014 hält und datumsmässig angibt, wann das Arbeitsverhältnis beginnt und wann es endet. Damit steht nicht der Arbeitsvertrag, wie er in Klagebeilage 3 vorliegt, sondern der GAV Solo 2014 und gegebenenfalls dessen Auslegung im Vordergrund der vorzunehmenden rechtlichen Beurteilung, ob ein befristetes oder unbefristetes Arbeitsverhältnis gegeben ist oder nicht.

Normative Bestimmungen eines GAV werden nach den Regeln der Gesetzesinterpretation ausgelegt (BGer. 4A_670/2010 E. 3.1; 4A_494/2007 E. 1.2; 4C.69/2006 E. 2.3; BGE 133 III 213 E. 4.2.; 127 III 318 E. 2a). „Ein Gesetz muss in erster Linie aus sich selbst heraus, das heisst nach Wortlaut, Systematik, Sinn und Zweck der Regelung verstanden werden. Auszurichten ist die Auslegung auf die ratio legis, die das Gericht allerdings nicht nach seinen eigenen, subjektiven Wertvorstellungen, sondern nach den Vorgaben und Regelungsabsichten des Gesetzgebers aufgrund der herkömmlichen Auslegungselemente zu ermitteln hat. Das Bundesgericht befolgt bei der Gesetzesauslegung einen pragmatischen Methodenpluralismus und lehnt es namentlich $\mathrm{ab}$, die einzelnen Auslegungselemente einer hierarchischen Prioritätsordnung zu unterstellen“ (BGE 142 III 102 E. 5 m.H.). Ein klarer, unzweideutiger Gesetzeswortlaut ist allerdings bindend, sofern er den wirklichen Sinn der Norm wiedergibt. Gegenteiliges kann sich aus der Entstehungsgeschichte der Norm, ihrem Zweck, aus dem Zusammenhang mit anderen Normen oder daraus ergeben, dass sie nicht dem Willen des Gesetzgebers entspricht (BGE 127 III 318 E. 2a). Auf einer Stufe damit steht auch eine allfällige rechtsvergleichende Auslegung (BGE 138 III 497 E. 3.4; siehe Ziff. 2.5.6.2.9.). Da die normativen Bestimmungen zwar eine gesetzesähnliche Funktion haben, aber durch Vertrag entstehen, ist ihre Auslegung auch von der Vertragsauslegung mitbestimmt (BGE 136 III 283 E. 2.3.1 = Praxis 2011 Nr. 29; 133 III 213 E. 5.2). Der Wille der Parteien des GAV als Auslegungselement ist daher stärker als jener des Gesetzgebers zu gewichten. Mit Rücksicht auf den Vertrauensschutz der an der Normsetzung nicht beteiligten Einzelvertragsparteien ist zu prüfen, ob sich der gemäss Vertragsauslegung ermittelte Vertragswille mit einer objektiven Auslegung nach Wortlaut, Sinnzusammenhang und Ratio verträgt (BGE 136 III 283 E. 2.3.1; 133 III 213 E. 5.2; BGer. 4C.216/2005 E. 2.1). Falls die normativen GAV-Bestimmungen lückenhaft sind, ist grundsätzlich wie bei Gesetzeslücken zu verfahren (BGE 133 III 213 E. 5.2).
2.5.5. Ausgangspunkt der Auslegung eines GAV ist dessen Wortlaut. Zwei Vorschriften des GAV Solo 2014 sprechen von der "Dauer" bzw. der "vereinbarten Zeit" des Arbeitsverhältnisses. Während dieser Ausdruck in Art. 7 GAV Solo 2014 sowohl eine befristete als auch eine unbefristete Vertragsdauer zulässt, ist er in Art. 8 GAV Solo 2014 (siehe Ziff. 2.5.3.) nur mit einer befristeten Dauer denkbar. Auf eine befristete Dauer weisen auch die Art. 9 GAV Solo 2014 in Verbindung mit dem zur Verwendung vorgeschriebenen Vertragsformular und Art. 29 GAV Solo 2014 hin, wenn sie von Spielzeiten sprechen, für die der Arbeitsvertrag abzuschliessen bzw. abgeschlossen worden sei. Hingegen wirft die Möglichkeit der Nichtverlängerungserklärung in Art. 29 GAV Solo 2014, ohne deren Ausübung der Vertrag für die nächste Spielzeit erneuert werde, Fragen auf. Während das Wort "Nichtverlängerung" auf die Ausdehnung einer vereinbarten Vertragsdauer Bezug nimmt, soll der Ausdruck "erneuern" für den Abschluss eines neuen Arbeitsvertrages für die Zeit nach Ablauf des Arbeitsvertrages für die abgelaufene Spielzeit stehen. Der Ausdruck erinnert an das Wort Neuerung (Novation), was die Tilgung einer alten Schuld durch Begründung einer neuen Schuld bedeutet (Art. 116 Abs. 1 OR). Hinzu kommt, dass die Nichtverlängerungserklärung ohne Mitwirkung der jeweils anderen Vertragspartei ausgeübt wird und damit wie die Kündigung als Gestaltungsrecht erscheint, sodass sich die Frage stellt, inwiefern sich die beiden Rechtsinstitute unterscheiden, damit sie nicht als gleichbedeutend gelten können und müssen. Es ergibt sich aus alledem, dass der Wortlaut von Art. 29 GAV Solo 2014 nicht als eindeutig und klar gelten kann. Die Vorschrift ist daher aufgrund der weiteren Auslegungselemente auszulegen.

2.5.6.1. In systematischer Hinsicht ist von Art. 8 GAV Solo 2014 und vom Vertragsformular, das gem. Art. 9 Abs. 1 GAV Solo 2014 obligatorisch ist, auszugehen. Der GAV Solo 2014 spricht darin von der "vereinbarten Zeit" bzw. vom "Spielzeitenvertrag". Es ist darunter, wie erläutert (s. Ziff. 2.5.5.), ein befristeter Arbeitsvertrag zu verstehen. Art. 7 GAV Solo 2014 lässt sich daher in systematischer Auslegung auf befristete Arbeitsverträge beschränken. Weniger klar ist die Situation bei Art. 29 GAV Solo 2014. Die Vorschrift greift den Ausdruck "Spielzeit", für die der Arbeitsvertrag erneuert werde, und damit den befristeten Spielzeitenvertrag auf. Ob die "Nichtverlängerungserklärung" in Art. 29 GAV Solo 2014 dazu führt, dass das Arbeitsverhältnis befristet bleibt oder aber zu einem unbefristeten wird, lässt sich aufgrund der systematischen Auslegung nicht abschliessend beantworten, weil die Regelung der Weiterführung des Arbeitsverhältnisses in Frage steht. Mit Hinweis auf Art. 334 Abs. 2 OR wäre eine Verlängerung auf unbestimmte Dauer eine der Spielzeitenverlängerung grundsätzlich gleichgestellte Möglichkeit.

2.5.6.2. Die Beklagte beruft sich darauf, dass die Befristung von Bühnenarbeitsverträgen auf eine oder mehrere Spielzeiten einen der wichtigsten Bühnenbräuche darstelle, der auch gewohnheitsrechtlich begründet sei. Art. 8 Abs. 2 GAV Solo 2014 rezipiere diesen gewohnheitsrechtlichen Bühnenbrauch [...]. Die Klägerin bestreitet, dass die Befristung in der Schweiz einen langjährigen Bühnenbrauch darstelle [...]. Beim System der 
Spielzeitenverträge handle es sich auch nicht (ausschliesslich) um eine Bühnenusanz, sondern diese seien im GAV Solo 2014 vorgesehen [...].

2.5.6.2.1. Beide Parteien anerkennen zu Recht die Existenz von Bühnenbräuchen bzw. -usanzen. Es handelt sich um im Bühnenleben übliches Verhalten bzw. entsprechende Handlungsweisen der Beteiligten, die allgemein beobachtet werden und damit herrschend sind (Margaretha Kugler Die Arbeitsbedingungen der Bühnenkünstler, insbesondere nach dem schweizerischen Gesamtarbeitsvertrag, 1959, S. 43; Stephan Wolf Berner Kommentar, Art. 1-9 ZGB, 2012, Art. 5 Rn. 100, S. 832). Gebräuche und Usanzen bestehen in vielen Geschäftsbereichen. Ihre Ausdehnung kann unterschiedlich sein, sich etwa auf einen einzigen Betrieb bzw. ein einzelnes Theater beschränken oder eine ganze Branche bzw. alle Bühnen umfassen (Kugler S. 43). Entsprechend wird von Betriebs- bzw. Branchenübung gesprochen. Derartige Übungen können bei der Auslegung und Ergänzung von Rechtsgeschäften zu berücksichtigen sein (BK-Wolf Art. 5 Rn. 106, S. 834; Reto Ruoss Einleitungsartikel des Schweizerischen Zivilgesetzbuches, 3. Aufl. 1979, S. 164; Kugler S. 43). Dort, wo das Gesetz auf eine Übung verweist, wird diese zur subsidiären Rechtsquelle (z.B. Art. 322 Abs. 1 OR: üblicher Lohn; Arnold Marti Zürcher Kommentar, Einl. Art. 1-7 ZGB, Art. 5 Rn. 217, S. 1050 und Rn. 238, S. 1057 f.; BK-Wolf Art. 5 Rn. 103, S. 833). Als Rechtsquelle dient eine Übung zudem, wenn sie die qualifizierten Voraussetzungen des Gewohnheitsrechts erfüllt (Susan Emmenegger/Axel Tschentscher Berner Kommentar, Art. 1 ZGB Rn. 424, S. 378; Ruoss S. 165). Nach Art. 1 Abs. 2 ZGB haben Gerichte Gesetzeslücken in erster Linie und soweit vorhanden mit Gewohnheitsrecht zu füllen. Gewohnheitsrecht setzt neben einer über lange Zeit eingehaltenen Rechtsregel - und damit Übung (longa consuetudo) - auch die bei den beteiligten Geschäftskreisen - in Bezug auf Arbeitsverhältnisse damit bei Arbeitgebern und Arbeitnehmern - herrschende Überzeugung voraus, dass es sich dabei um eine rechtsverbindliche Regel handelt (opinio necessitatis bzw. iuris). Um als Bundesgewohnheitsrecht gelten zu können, muss die betreffende Regel nach herrschender Ansicht zudem einen gemeinschweizerischen Charakter aufweisen (BK-Emmenegger/Tschentscher Art. 1 ZGB Rn. 418 f., S. 376). Übungen bzw. Usanzen sind soziale Normen und damit Tatsachen, die durch diejenige Partei zu beweisen sind, die sich darauf beruft (Art. 8 ZGB; BGer. 4C.92/2006 E. 1.3.1.; Hans Peter Walter Berner Kommentar, Art. 8 ZGB Rn. 111, S. 1092). Hingegen erfolgt die Feststellung und Anwendung einer Übung, auf die das Gesetz verweist, sowie von lückenfüllendem Gewohnheitsrecht von Amtes wegen durch die Gerichte (BKWolf Art. 5 N 125, S. 841; BK-Walter Art. 8 ZGB N 109, S. 1091).

Bühnenbräuche können zur Entstehung von normativen Vorschriften eines Gesamtarbeitsvertrages beitragen und darin eigentlich festgeschrieben werden. Daraus lässt sich auf den Vertragswillen der Gesamtarbeitsvertragsparteien bei der Vereinbarung der betreffenden gesamtarbeitsvertraglichen Bestimmungen schliessen. Zu untersuchen ist, ob und gegebenenfalls seit wann der Spielzeitenvertrag mit Nichtverlänge- rungserklärung im Sinne eines befristeten Vertrages als schweizerischer Bühnenbrauch gelten kann. An den Anfang ist dabei die Entwicklung in Deutschland zu stellen, die wie sich zeigen wird, einen bestimmenden Einfluss auf das schweizerische Bühnenrecht hatte.

2.5.6.2.2. In Deutschland hat der zwischen Theatern und Bühnenmitgliedern abgeschlossene Spielzeitenvertrag eine lange Tradition und gilt als Bühnenbrauch bei unklarer Vertragsdauer (Bernhard Riepenhausen Das Arbeitsrecht der Bühne, 2. Aufl., 1956, S. 7 Fn. 2 m.H.; Joachim Rübel Geschichte der Genossenschaft Deutscher Bühnen-Angehörigen (GDBA), 1989, S. 280). Angeführt werden verschiedentlich Bühnenengagementsverträge am Kürfürstlich-pfälzischen Theater in Mannheim aus dem Jahre 1795, die befristet waren und vorsahen, dass der Vertrag stillschweigend um ein Jahr verlängert werde, wenn ein halbes Jahr vor Ablauf der Vertragsdauer keine Aufkündigung erfolge, was auch für die folgenden Jahre gelte (Manfred Rehbinder Zur rechtsgeschichtlichen Entwicklung des Bühnenengagements, UFITA 1975, S. 87 ff., S. 97 f.; Bernhard Opolony Die Nichtverlängerungsmitteilung bei befristeten Bühnenarbeitsverhältnissen, Neue Zeitschrift für Arbeitsrecht, NZA, 2001, S. 1351 ff., S. 1351 f.; ders. Die Befristung von Bühnenarbeitsverhältnissen, Zeitschrift für Arbeitsrecht, ZfA, 2000, S. 179 ff., S. 185 ff.; Kurz / Kehrl / Nix, Praxishandbuch Theater- und Kulturveranstaltungsrecht, 2. Aufl. 2015, S. 29 Rn. 51). Auch als Johann Wolfgang Goethe Theaterleiter in Weimar war, schloss er mit den Schauspielern gleichlautende, zeitlich befristete Bühnenengagementsverträge ab. Darin war jeweils bestimmt, dass diejenige Partei, die den Vertrag nicht über das vorgesehene Ende an Ostern hinaus verlängern wollte, dies der anderen Partei bis Weihnachten anzeigen musste. Widrigenfalls werde der Vertrag um die gleiche Dauer als erneuert und verlängert angesehen (Arthur Rosenmeyer Der Weimarer Theatervertrag zur Zeit der Intendanz Goethes, UFITA 1932, S. 329 ff., S. 331 f.). Aus diesen Vorläufern entstand in Deutschland allmählich eine Bühnenusanz, die im Jahre 1926 weit verbreitet, aber noch nicht herrschend war, wie sich aus einem damaligen Rundschreiben des Deutschen Bühnenvereins ergibt. Durch das Bühnenoberschiedsgericht wurde jedoch im Jahre 1937 die rechtzeitige Mitteilungspflicht als feststehender, gewohnheitsrechtlicher Bühnenbrauch bezeichnet (Riepenhausen S. 7, 162; vgl. Rübel S. 280). Aufgrund dieses Bühnenbrauches haben die Tarifvertragsparteien im Jahre 1947 den Tarifvertrag über die Mitteilungspflicht geschlossen (Opolony NZA 2001, S. 1351 f.; ders. ZfA 2000, S. 187; Manfred Rehbinder Zum Zeitvertrag im Bühnenarbeitsrecht, UFITA 1980, S. 89 ff, S. 91), die im Jahre 1952 durch das Bühnenoberschiedsgericht "zu einer unabdingbaren Tarifnorm erhoben wurde" (Rübel S. 281). Der Normalvertrag regelt die Nichtverlängerungsmitteilung für die Soloverträge in § $61 \mathrm{NV}$, für die Chorverträge in $\S 83 \mathrm{NV}$ und für die Tanzverträge in $\S 96$ NV. Nach ständiger Rechtsprechung des Bundesarbeitsgerichts, des Bundesverwaltungsgerichts und des Bühnenoberschiedsgerichts ist die Nichtverlängerungsmitteilung keine Kündigung und kann ihr auch nicht gleichgestellt werden (BAG 23.10.1991 - 7 AZR 56/91 E. 4a m.H., BAG 28.10.1986 - 1 ABR 16/85 E. 2 m.H.; 
BOSchG 36/1993, 01/1996, 23/1997, www.buehnengenossenschaft.de; Nix/Hegemann/Hemke, § 61 Rn. 31; Hermann Josef Fischer/Steven A. Reich (Hrsg.) Der Künstler und sein Recht, Ein Handbuch für die Praxis, 3. Aufl. 2014, § 10 Rn. 130, S. 257 f.; Nele Urban Die Beendigung von Bühnenengagementsverträgen im Spannungsfeld von Kunstfreiheit und Arbeitnehmerschutz in Deutschland und Frankreich, 2005, S. 67 ff. m.H. in Fn. 287). Im Unterschied zur Kündigung beende sie nicht das Arbeitsverhältnis, sondern bestätige lediglich, dass die von Theater und Bühnenmitglied konkret vereinbarte Vertragsdauer nicht über diesen Zeitraum hinaus verlängert werden soll (BAG 23.10.1991 - 7 AZR 56/91 E. 4a m.H.; Kurz/Kehrl/Nix, S. 444 Rn. 6; Schaub/ Koch/Linck/Treber/Vogelsang Arbeitsrechtshandbuch, 16. Aufl., 2015, § 186 Rn. 8., S. 2011; Opolony NZA 2001, S. 1352). Obwohl die Rechtsnatur der Nichtverlängerungsmitteilung im deutschen Bühnenrecht nicht unumstritten ist, spricht ihr die herrschende Meinung in Literatur und Rechtsprechung die Qualität einer Willenserklärung ab und qualifiziert sie als reine Wissenserklärung (Urban S. 67 m.H. Opolony ZfA 2000, S. 188).

2.5.6.2.3. In der Schweiz kamen stehende Bühnen im 19. Jahrhundert auf (Paul Lang Bühnen und Drama der deutschen Schweiz im XIX. und beginnenden XX. Jahrhundert, 1924, S. 14; W. Bickel Auf der Bühne - und im Leben, Zur sozialen Lage der schweizerischen Bühnenkünstler, 1944, S. 8). Private Gesellschaften gründeten in verschiedenen Städten eigentliche Berufstheater (Lang S. 14 ff.; Bickel S. 5 f.; Stefan Koslowski Stadttheater contra Schaubuden, Zur Basler Theatergeschichte des 19. Jahrhunderts, 1998, S. 13). Es waren reine Geschäftstheater, die die Gesellschaften zunächst verpachteten, später in eigener Regie führten und die sich allmählich zu eigentlichen Kulturtheatern entwickelten (SBV, Subventionsgesuch an den Bundesrat von 1921, S. 6, 48 f.; Bickel S. 8 f.; Kugler S. 11 f.; Christoph Kohler Wozu das Theater, Zur Entstehungsgeschichte der Theatersubventionen in Zürich (1890-1928), 2008, S. 69). Die Theatereigner und -betreiber hatten stets mit wirtschaftlichen Schwierigkeiten zu kämpfen. Um diese zu meistern, ersuchten sie die Gemeinwesen um Subventionen (Bickel S. 9; Kugler S. 11 f.; Koslowski S. 19 ff., 52 ff.; Kohler S. 60 ff.; SBV, Subventionsgesuch, S. 48, 51) sowie um Schutz vor Konkurrenz durch andere darstellende Attraktionen, wie sie von den Zirkussen und später auch von den Lichtspiel- und Varietés-Theatern geboten wurden. Als eine der Ursachen für die wirtschaftlichen Schwierigkeiten erwies sich insbesondere der Aufstieg vom Geschäftstheater zum Kulturtheater (SBV, Subventionsgesuch, S. 48), das nur mit einem grösseren Aufwand betrieben werden konnte. Die beschränkten Mittel, über die die Theaterbetreiber verfügten, führten dazu, dass diese die Bühnenkünstler zu geringen Gagen und fast ausschliesslich jeweils für die Dauer einer Spielzeit anstellten. Folge davon war die jährliche Erneuerung der Anstellung mit denjenigen Bühnenkünstlern, die die Zusage erhielten, auch in der darauf folgenden Saison am Theater mitzuwirken. Da die Spielzeiten oft nur sechs, später acht bis neun Monate dauerten (vgl. Koslowski Tabelle 2 S. 190 f.: zur Spielzeitendauer am Basler Stadttheater in den Jahren 1834 bis 1904), waren die Bühnenkünstler bis zum Saisonbeginn bis zu einem halben Jahr ohne
Arbeit und Lohn, falls sie für die Zwischenzeit, regelmässig im Sommer, kein weiteres Engagement fanden (SBV, Subventionsgesuch, S. 6 f.; Bickel S. 9; Schweizer Bühne, Oktober 1931, S. 2). Die Forderung der Bühnenkünstler nach Jahresverträgen war deshalb alt (Bickel S. 21; Schweizer Bühne, Juni 1931, S. 4). Selbst der schweizerische Bühnenverband bezeichnete Jahresverträge als nützlich und notwendig, als er im Jahre 1921 mit einem Subventionsbegehren an den schweizerischen Bundesrat gelangte. Durch dauernde Verträge würden die Künstler innerlich an die Theater gefesselt. Eine Verbesserung ihrer ökonomischen Lage verleihe ihnen zudem die zur Ausübung ihrer künstlerischen Tätigkeit erforderlichen Sicherheit (SBV, Subventionsgesuch, S. 50). Allerdings verhinderten die ungünstigen finanziellen Verhältnisse an den Theatern weiterhin und für lange Zeit die Verwirklichung dieses Zieles. Noch für das Jahr 1944 wird berichtet, dass die Spielzeiten an einigen Theatern zwar verlängert worden seien. Ein ganzes Jahr würden sie aber nur gerade in Zürich und St. Gallen dauern (Bickel S. 22). Es waren denn auch für lange Zeiten nur zwei Theater, die ganzjährig waren (Schweizer Bühne, Oktober 1931, S. 2). Es ist damit festzustellen, dass Spielzeitenverträge an schweizerischen Berufstheatern seit dem 19. Jahrhundert gebräuchlich sind.

2.5.6.2.4. Für die Spielzeitenverträge als Bühnenbrauch an schweizerischen Theatern wird ferner auf die von Goethe in Weimar entwickelten Regeln der Theaterproduktion und -veranstaltung verwiesen. Sie seien typisch für das heutige Ensembletheater mit seinen Spielzeitenverträgen, den Anspruch auf Beschäftigung, die Treue- und Mitwirkungspflicht der Bühnenmitglieder gegenüber der Intendanz und delegationsweise gegenüber der Regie sowie für das System der Nichtverlängerungserklärung (Peter Mosimann Goethes Intendanz, die Bühnenbräuche und das heutige Theaterrecht, Festschrift JeanFritz Stöckli, 2014, S. 389 ff., S. 397 ff.).

Typischer Eckpfeiler des Spielzeitenvertrages sei der Beschäftigungsanspruch seit Anfang des 19. Jahrhunderts (Mosimann FS Stöckli, S. 397). Ein solcher Anspruch war von Goethe jedoch noch nicht anerkannt gewesen (Rosenmeyer S. 340). Ansätze für eine Anerkennung des Anspruchs im deutschen Theaterrecht gingen auf die französische und italienische Rechtsprechung und Rechtslehre zurück, wo die grundlegende Bedeutung des Beschäftigungsrechts bereits in der Mitte des 19. Jahrhunderts anerkannt war und Eingang in die Praxis gefunden hatte (Jürgen Frank Der Beschäftigungsanspruch und die soziale Gleichbewertung der individuellen Arbeitsleistung aus der Sicht der besonderen Interessenlage im Bühnenvertragsrecht, 1963, S. 15 f. m.H.). In der deutschen Rechtsprechung wurde er erstmals im Jahre 1910 vom Reichsgericht bejaht, dem sich später auch die Bühnenschiedsgerichte anschlossen (Frank S. 17; Dienstag/Elster S. 437 f. m.H.; Rehbinder UFITA 1975, S. 103). In der Folge wurde der Anspruch der Bühnenmitglieder auf eine angemessene Beschäftigung im Normalvertrag zum deutschen Tarifvertrag von 1919 verankert (NV § 5). Der Anspruch der Bühnenmitglieder auf 
eine angemessene Beschäftigung fand auch Eingang in den Anstellungsvertrag zum GAV Solo (AV Ziff. V zum GAV Solo 1937) und gilt noch heute in gleicher Weise (Art. 17 GAV Solo 2014).

Der Anspruch hat seine Begründung darin, dass eine tatsächliche Beschäftigung dem Bühnenkünstler zum Erhalt seiner Berufsfähigkeit und Spielpraxis und zur künstlerischen Weiterentwicklung dient. Der Bühnenkünstler ist auf die Möglichkeit angewiesen, vor Publikum aufzutreten, Anerkennung für seine künstlerische Gestaltung zu erlangen, mit seinem Namen bekannt zu werden und das Selbstvertrauen in seine Fähigkeiten zu bewahren (Frank S. 16 m.H.; Kugler S. 72 m.H.; Dienstag/Elster S. 439). Eine angemessene Beschäftigung wird als Grundlage zur Existenzsicherung im Engagementswechsel beim Zeitvertrag angesehen (Bernhard Riepenhausen Grundzüge des Arbeitsrechts der Bühnenkünstler, UFITA 1957, S. 27 ff., 51 f.). Ausfluss des Beschäftigungsanspruchs ist in Art. 29 Abs. 3 GAV Solo 2014 denn auch die Einschränkung der Nichtverlängerung eines Bühnenengagementsvertrages durch ein Theater in Zusammenhang mit einem Direktionswechsel. Falls ein Bühnenengagement in einer Spielzeit vor oder nach einem Direktionswechsel nicht verlängert werden soll, muss der gewählte Direktor das betreffende Bühnenmitglied nachweislich in zwei repräsentativen Rollen bzw. Partien gesehen haben. Der Beschäftigungsanspruch hat damit zweifellos zur Verfestigung des Spielzeitenvertrages im Bühnenbetrieb beigetragen.

Ferner wird der Spielzeitenvertrag als im Interesse des Regietheaters mit Ensemble typisch bezeichnet (Mosimann FS Stöckli, S. 398 ff.; ders. Bühnenarbeitsrecht, S. 683 Rn. 130). Das Ensembletheater baut grundsätzlich auf eine längerfristige Zusammenarbeit zwischen Theater und Bühnenkünstlern. Andererseits ist beim Regietheater die Bühnenleitung darauf angewiesen, mit Bühnenkünstlern zusammenzuarbeiten, die die Regiekonzeption am besten umzusetzen imstande sind (Manfred Rehbinder UFITA 1980, S. 100). Dies zeigt sich ganz besonders beim Wechsel der Intendanz. Diesem Zusammenspiel zwischen Konstanz und erforderlicher Veränderung des Ensembles kommt der Spielzeitenvertrag auf ideale Weise entgegen.

2.5.6.2.5. Es wurde erwähnt, dass der Spielzeitenvertrag in Deutschland eine lange Tradition hat. Das Theaterwesen in der Schweiz stand im 19. und bis ins 20. Jahrhundert hinein unter einem starken deutschen Einfluss. Nicht nur an den grösseren Theatern, sondern auch an den Bühnen kleinerer Kantonshauptstädte waren in der Regel für den Theaterbetrieb deutsche Geschäftsdirektoren verantwortlich, die ihre Ensembles fast ausschliesslich aus deutschen und österreichischen Bühnenmitgliedern rekrutierten (Lang S. 31; Koslowski S. 12 f.; Schweizer Bühne, Oktober 1931, S. 3). Es ist daher nicht erstaunlich, dass die Schweiz in Theaterdingen bis in die frühen 30er Jahre des letzten Jahrhunderts als deutsche Provinz galt (Lang S. 25). Die schweizerischen Berufstheater waren dabei in ihrer Gangart völlig nach Berlin ausgerichtet und vom deutschen Theater abhängig (Bickel S. 5; Kugler S. 30). Für den deutschen Einfluss legt auch die Nähe der Bühnenkünstlerverbände in
Deutschland und der Schweiz zueinander ein Zeugnis ab. Der im Jahre 1920 gegründete "Verband der Bühnenkünstler in der Schweiz" war eine Sektion der Genossenschaft deutscher Bühnenangehöriger (GDBA), bis er im Jahre 1931 als Schweizerischer Bühnenkünstlerverband zum VPOD stiess (SBV, 50 Jahre Schweizerischer Bühnenverband 1920-1970, S. 58; Kugler, S. 30; SBV, Subventionsgesuch S. 49), wo er bis im Jahre 1995 blieb. Noch heute ist er mit der GDBA der Vereinten Dienstleistungsgewerkschaft Deutschlands (ver.di) und der Sektion Bühne der österreichischen Gewerkschaft Kunst, Medien, Sport und freie Berufe (kmsfb) zum Kartellverband deutschsprachiger Bühnenangehöriger zusammengeschlossen. Auch der Verband schweizerischer Bühnen, den die Stadttheater von Basel, Bern, Luzern, Genf, St. Gallen und Zürich sowie das Corsotheater in Zürich 1920 gründeten, stand unter dem Druck ausländischer Theaterorganisationen, von dem er sich auch zu befreien versuchte, um dem Theaterkunstbetrieb in der Schweiz ein mehr nationales Gepräge zu geben (SBV, Subventionsgesuch, S. 49 f.).

Die neu gegründeten Arbeitgeber- und Arbeitnehmerverbände versuchten schon bald in einem ersten Gesamtarbeitsvertrag für die Solisten von 1923 ihren Mitgliedern bessere Arbeits- und Existenzbedingungen zu verleihen und unsoziale Bestimmungen aus den überlieferten Bühnenengagementsverträgen zu eliminieren (Bickel S. 11). Aufgrund der engen Abhängigkeit des schweizerischen vom deutschen Theaterwesen wurde der wenige Jahre zuvor in Kraft getretene deutsche Tarif- und Normalvertrag von 1919 herangezogen (SBV, 50 Jahre, S. 55). Der Tarifvertrag hatte Geltung für das Deutsche Reich, führte aber bezeichnenderweise gleichzeitig an, dass eine Ausdehnung auf Österreich und die Schweiz vorbehalten bleibe (Tarifvertrag 1919, Ziff. I). Allerdings liessen sich die deutschen Verhältnisse nicht einheitlich auf die Schweiz übertragen (SBV, 50 Jahre, S. 55), sodass, wie der Bühnenkünstlerverband feststellte, "manche Bestrebungen der Bühnenkünstler" im Gesamtarbeitsvertrag nicht verwirklicht werden konnten (Bickel S. 11). Der Gesamtarbeitsvertrag für die Solisten von 1923 wurde in den folgenden Jahrzehnten zwischen den Verbänden immer wieder neu verhandelt, abgeschlossen und in Kraft gesetzt, so in den Jahren 1930, 1937, 1946 (SBV, 50 Jahre, S. 57 ff.), 1967, 1981 (ergänzt im Jahre 1997) sowie 2014.

2.5.6.2.6. Integrierender Bestandteil der Gesamtarbeitsverträge für die Solisten war und ist noch heute das Formular für den Anstellungsvertrag zwischen Bühne und Bühnenmitglied, das zum Abschluss eines Bühnenengagements zu verwenden ist, um die Vereinbarung nichtiger Vertragsabreden zu vermeiden (Art. 9 GAV Solo 2014). Während die normativen Bestimmungen - wie beim deutschen Normalvertrag (NV; Tarifvertrag 1919 Ziff. III.) - zunächst (bis und mit GAV Solo 1937) im Formular des Anstellungsvertrages enthalten waren, sind sie seither (seit GAV Solo 1946) im GAV-Dokument selber zu finden. Hingegen war und ist noch im Anstellungsformular - wie auch im deutschen Normalvertrag ( $\$ 2$ NV von 1919) - stets vorgesehen, dass das Vertragsverhältnis an einem zwischen Bühne und Bühnenmitglied individuell vereinbarten, mit Datum festgelegten Tag 
beginnt und mit Ablauf eines gleichermassen bestimmten Tages endet. Damit wurde von Beginn weg der Spielzeitenvertrag, wie er an den schweizerischen Berufstheatern schon vorher regelmässig abgeschlossen worden war, als fester Bestandteil auch im Gesamtarbeitsvertrag verankert. Dass der Spielzeitenvertrag als zeitlich befristeter Anstellungsvertrag die Regel war, zeigt sich darin, dass die Gesamtarbeitsvertragsparteien es den Bühnen und Bühnenmitgliedern überliessen, davon im Einzelfall abzuweichen und eine Kündigungsfrist vorzusehen. Für diesen Fall sah der Anstellungsvertrag vor, dass die zu vereinbarenden Kündigungsfristen für beide Parteien gleich lang gewählt werden mussten (Anstellungsvertrag Ziff. VIII. zum GAV Solo 1937). Wie sich aus einem Briefwechsel zwischen dem SBKV und dem Vorstand der Genossenschaft des Stadttheaters Basel im Januar 1941 ergibt, hatte der Genossenschaftsvorstand schon seit einigen Jahren jeweils anfangs Januar über die Reengagements oder die Nichtreengagements der bisherigen Bühnenmitglieder (Staatsarchiv Basel-Stadt) beschlossen. In den Verhandlungen der Verbände über den GAV Solo 1946 schlug der SBKV im Abschnitt "C. Beendigung des Dienstvertrages" einen Art. 27 mit folgendem Wortlaut vor, der zwei Absätze umfassen sollte:

\section{„Art. 27 Kündigung}

${ }^{1}$ Ist ein Dienstvertrag auf unbestimmte Zeit abgeschlossen, so kann er mangels anderer Abrede im Dienstvertrag beidseitig jeweils nur bis spätestens am 1. Januar auf das Ende der Spielzeit gekündigt werden.

${ }^{2}$ Wird im Dienstvertrag eine andere Kündigungsfrist vereinbart, so muss sie für beide Teile gleich sein. Bei Ungleichheit der Frist gilt für beide Teile die längere Frist."

Es lag darin offensichtlich das Bestreben, den Spielzeitenvertrag möglichst durch einen auf unbestimmte Dauer abgeschlossenen Dienstvertrag abzulösen, um im Interesse der Bühnenkünstler längerfristige Arbeitsverhältnisse zu schaffen. Triebfeder dazu konnte nicht ein irgendwie gearteter Kündigungsschutz gewesen sein, der damals weder im schweizerischen Recht existierte noch mit dem neuen GAV Solo geschaffen werden sollte. Man kannte jedoch zweifellos die in Deutschland vertretene Rechtslehre, wonach die Mitteilung, einen Arbeitsvertrag nicht über das vereinbarte Ende hinaus andauern zu lassen, als Kündigung zu werten war. Da die massgebende Rechtsprechung in Deutschland dieser Ansicht jedoch nicht folgte, lag es nahe, die Kündigung im GAV festzuschreiben, um derartigen Auslegungen zuvorzukommen. Der SBV hingegen lehnte, wie sich aus den Vertragsentwürfen vom 19. April 1943 und 6. Mai 1943 ergibt, die erhalten sind (Staatsarchiv Basel-Stadt), diesen Vorschlag konsequent ab. Im definitiven GAV, auf den sich der SBV und der SBKV einigten, lautete im Abschnitt "C. Beendigung des Dienstvertrages" die umstrittene Vorschrift, die zum Art. 25 GAV Solo 1946 geworden war, in einem einzigen Absatz wie folgt:

\section{„Art. 25 Kündigung}

Falls eine der Vertragsparteien bis zum 31. Januar nicht die Erklärung abgibt, dass der Vertrag nicht erneuert werde, gilt er als für die nächste Spielzeit erneuert."

Der GAV Solo 1946 folgte darin dem erwähnten, an deutschen Bühnen damals schon herrschenden Bühnenbrauch (siehe Ziff. 2.5.6.2.2.) einer Nichtverlängerungsmitteilung. Das Kündigungsrecht wurde lediglich im Formular des Anstellungsvertrages als Variante bei Verträgen über mehrere Spielzeiten, wie sie Art. 26 des GAV Solo 1946 („Kündigung gegenüber Anfängern“) bei Anfängern vorsah, oder bei Verträgen auf unbestimmte Dauer aufgeführt. Dieser Bühnenbrauch bezüglich der Nichtverlängerungserklärung hatte im Zuge des deutschen Einflusses auf das schweizerische Theaterwesen auch die hiesigen Bühnen erreicht und die Bühnenverhältnisse massgeblich beeinflusst. Zeuge davon ist der angeführte Briefwechsel zwischen dem SBKV und dem Vorstand der Genossenschaft des Stadttheaters Basel. Teil dieses Bühnenbrauchs waren Anhörungs- und Konsultationsrechte, die dem Personal an den Theatern zugestanden wurden. An den Sitzungen der Verwaltung des Basler Stadttheaters nahm der vom Personal gewählte Personalvertreter mit beratender Stimme teil. Dies galt insbesondere auch für die Sitzungen, an denen die Theaterverwaltung die Reengagements und Nichtreengagements diskutierte und darüber entschied. Zudem fanden fallweise Gespräche zwischen der Theaterverwaltung und Delegationen aus dem Personal oder dem SBKV bezüglich solcher Entscheide statt (Briefwechsel zwischen SBKV und dem Genossenschaftsvorstand des Basler Stadttheaters vom 10. und 16.1.1941; Schreiben des SBKV vom 24.1.1950 an den Verwaltungsratspräsidenten der Genossenschaft des Basler Stadttheaters; Staatsarchiv Basel-Stadt).

2.5.6.2.7. Die Nichtverlängerungserklärung wurde auch in den nachfolgenden Gesamtarbeitsverträgen beibehalten, jedoch detaillierter geregelt. Insbesondere wurde im Gesamtarbeitsvertrag von 1981 der Zeitpunkt, bis zu welchem die Nichtverlängerungserklärung spätestens abzugeben war, mit Ausnahme des ersten Vertragsjahres vom 31. Januar auf den 31. Oktober vorverlegt (Art. 29 Abs. 1 GAV Solo 1981). Dies geschah nicht zuletzt deshalb, um den Bühnenkünstlern für ihre Suche nach einem neuen Engagement zeitlich entgegenzukommen. Eine solche Vorverlegung wurde bereits in früheren Jahren an einzelnen Bühnen, wenn besondere Umstände vorlagen, fallweise gewährt (Briefwechsel zwischen SBKV und Genossenschaft des Basler Stadttheaters vom 9. und 17.12.1954).

Unverändert seit dem GAV Solo 1946 blieb hingegen, dass die Vorschrift über die Nichtverlängerungserklärung auch im GAV Solo aus dem Jahre 1981 weiterhin mit „Kündigung" überschrieben war. Das Appellationsgericht Basel-Stadt hatte im Jahre 1994 die analoge Vorschrift über die Nichtverlängerungserklärung des Gesamtarbeitsvertrages für das künstlerische Chor- und Gruppenpersonal aus dem Jahre 1982 zu beurteilen. Dabei ging es davon aus, dass der Ausdruck "Kündigung" 
im Titel von Art. 29 des GAV Chor 1982 von den GAV-Parteien aus Nachlässigkeit aus früheren Gesamtarbeitsverträgen übernommen worden sei (AGE vom 13.10.1994, Jahrbuch des schweizerischen Arbeitsrechts, JAR 1995, S. 138 E. 2), wobei es sich hierzu auf Erkenntnisse aus der Rechtslehre stützte, die die Nichtverlängerungserklärung nicht als Kündigung werten (Elisabeth Anne Schellenberg Der Bühnenengagementsvertrag im schweizerischen Recht, Unter besonderer Berücksichtigung der Gesamtarbeitsverträge für die deutsche Schweiz, 1990, S. 78; Manfred Rehbinder Mitteilung des Instituts für Schweizerisches Arbeitsrecht, ArbR, 1991, S. 142). In der Folge änderten der SBV und der SBKV die Gesamtarbeitsverträge in dieser Hinsicht und überschrieben den Art. 29 GAV mit „Nichtverlängerungserklärung" (GAV Solo vom 5.6.1981 bzw. GAV Chor und Ballett vom 6.9.1982, jeweils in der Fassung vom 3.12.1997).

Änderungen eines Gesamtarbeitsvertrages kommen wie der ursprüngliche Vertragsabschluss durch übereinstimmende Willenserklärungen der vertragsschliessenden Verbände zustande. Einem solchen Vertragswillen kommt bei der Auslegung des GAV eine im Vergleich zum gesetzgeberischen Willen verstärkte Bedeutung zu (siehe Ziff. 2.5.4.). Er ist daran zu messen, ob er sich mit einer objektiven Auslegung nach Wortlaut, Sinnzusammenhang und Ratio verträgt (BGE 133 III 213 E. 5.2).

Vorliegend könnte die Änderung des GAV Solo hinsichtlich des Vertragswillens der beiden Verbände auf zwei Arten zu interpretieren sein. Es könnte sich bloss um eine sprachliche Vereinheitlichung innerhalb der betreffenden Vorschrift handeln, um in der Überschrift den gleichen Ausdruck wie in der Regelung der Vorschrift selber zu verwenden, ohne dass damit eine Aussage verbunden wäre, wie die Bezeichnung rechtlich zu charakterisieren ist. Andererseits könnte die Änderung der Überschrift darüber hinaus zum Ausdruck bringen, dass die in Art. 29 GAV Solo 2014 geregelte Nichtverlängerungserklärung nach dem Willen beider Gesamtarbeitsvertragsparteien in ihren Wirkungen nicht mit einer Kündigung gleichzusetzen ist. Auf das Erstere deutet hin, dass die Änderung unter den beiden Verbänden umstritten war und nicht, wie bei früheren GAVÄnderungen, von klärenden interpretativen Notizen begleitet ist. Für das Zweitere spricht hingegen, dass die Änderung offensichtlich im Nachgang zum erwähnten Entscheid des Appellationsgerichtes ergangen war, der von einer Nachlässigkeit bei der Verwendung des Wortes Kündigung im GAV Chor 1982 ausging, die Frage der rechtsgestaltenden Wirkung der Nichtverlängerungserklärung jedoch ausdrücklich offenliess. Dies erzeugte das Bedürfnis, im Sinne der Rechtssicherheit für Klarheit zu sorgen. Ein eindeutiges Resultat, wieweit der Wille der Verbände zu verstehen ist, den Gesamtarbeitsvertrag abzuändern, lässt sich unter diesen Verhältnissen aus der Änderung selber nicht herauslesen. Letztlich liegt die Antwort auf diese Auslegungsfrage in der Auslegung von Art. 25 GAV Solo 1946 und stellt nur den Nachvollzug dessen dar, was damals geschaffen und in der Folge beibehalten wurde.
2.5.6.2.8. Die Nichtverlängerungserklärung entwickelte, wie sich zeigte, sich auf der Grundlage eines zeitlich auf eine Spielzeit beschränkten Anstellungsvertrages zwischen Theater und Bühnenmitglied. Der Spielzeitenvertrag hatte sich als Bühnenbrauch etabliert, der zunächst im Formularvertrag zum GAV und später im Gesamtarbeitsvertrag selber als Regelfall des Anstellungsvertrages verankert wurde. Die Mitteilung, den Spielzeitenvertrag nicht zu verlängern, bevor dieser ausläuft, wurde, nachdem er längere Zeit an den Berufstheatern gebräuchlich war, ebenfalls im Gesamtarbeitsvertrag festgeschrieben. Das System der Nichtverlängerungserklärung hatte sich dabei in den Gesamtarbeitsverhandlungen gegen eine als solche deklarierte Kündigungsregelung durchgesetzt. Allerdings geschah dies vor dem Hintergrund, dass das schweizerische Arbeitsvertragsrecht damals noch keinen Kündigungsschutz kannte. Im Obligationenrecht traten der zeitliche Kündigungsschutz im Jahre 1972 und der missbräuchliche Kündigungsschutz [Schutz gegen missbräuchliche Kündigungen] im Jahre 1988 in Kraft. In der Folge wurden im GAV Solo weder das System der Spielzeitenverträge und der Nichtverlängerungserklärung geändert noch eine generelle ordentliche Kündbarkeit der Bühnenengagementsverträge eingeführt. In die Zeit nach 1988 fällt vielmehr bloss die auszulegende Änderung der Überschrift des Art. 29 GAV Solo 2014 von „Kündigung“ in „Nichtverlängerungserklärung".

Dieser Vertragswille (siehe Ziff. 2.5.4.) hält einer objektiven Auslegung nach Wortlaut, Sinnzusammenhang und Ratio stand. Sowohl der Wortlaut von Art. 29 GAV Solo 2014 als Ganzes, der Sinnzusammenhang zwischen dem Spielzeitenvertrag, wie er in mehreren Vorschriften im Gesamtarbeitsvertrag verankert ist, und der Nichtverlängerungserklärung sowie die Ratio von Art. 29 GAV Solo 2014, wie sie aus ihrer Entstehungsgeschichte und ihrer Anwendung zum Ausdruck kommt, zeigen dies auf. Daraus ergibt sich der Schluss, dass der eingeführte gesetzliche Kündigungsschutz keine Anwendung auf sachlich gerechtfertigte befristete Bühnenengagementsverträge findet. Hinzuweisen ist dabei auch auf ein Urteil des Appellationsgerichtes Basel-Stadt im Jahre 1998, das die Erklärung der Nichtverlängerung nach Art. 29 Abs. 1 GAV Solo 1981 - in Abweichung zum Urteil von 1994 (siehe Ziff. 2.5.6.2.7) und zu einem weiteren, späteren Urteil vom 29.2.2000 (Verf. Nr. 1020/1999), wo es diese Frage beide Male explizit offenliess - zwar als Kündigungserklärung interpretierte, gleichzeitig in einem obiter dictum jedoch betonte, dass angesichts einer damals anwendbaren achtmonatigen und damit aussergewöhnlich langen Kündigungsfrist sich „ein besonderer, namentlich zeitlicher Kündigungsschutz kaum rechtfertigen" lasse (AGE vom 14.7.1998, Verf. Nrn. 1000-1003/1997, E. 2c, S. 6).

Dem Bühnenschiedsgericht ist allerdings bekannt, dass in Härtefällen, die sich aus der Nichtanwendbarkeit des Kündigungsschutzes, insbesondere von Art. 336c OR, bisweilen ergaben, involvierte Bühnen und betroffene Bühnenmitglieder, unterstützt durch den SBKV, regelmässig nach Lösungen gesucht und solche gefunden haben, um finanzielle Einbussen abzufe- 
dern. Daraus ist allerdings kein Rechtsanspruch entstanden, der vom Bühnenmitglied oder vom SBKV geltend gemacht werden könnte. Diese Praxis entsprang vielmehr dem sozialpartnerschaftlichen Gedanken und wurde bis heute immer wieder angewandt. Auch im vorliegenden Fall wurden nach den Ausführungen der Parteien zwischen der Beklagten und dem SBKV vor Einreichung der Klage, denn auch, wenn letztlich doch aber erfolglos, Gespräche über eine gütliche Regelung geführt.

2.5.6.2.9. Die vorstehende Darstellung des Spielzeitenvertrages mit Nichtverlängerungserklärung als eine von der Kündigung verschiedene Rechtsfigur im deutschen Bühnenarbeitsrecht und deren Bedeutung für das Bühnenarbeitsrecht in der Schweiz ist auch eine rechtsvergleichende Auslegung schweizerischer Normen bzw. gesamtarbeitsvertraglicher Vorschriften. Gemäss Bundesgericht steht die rechtsvergleichende Auslegung im Rahmen des pragmatischen Methodenpluralismus (siehe Ziff. 2.5.4.) auf einer Stufe mit der systematischen, teleologischen und historischen Auslegung (BGE 138 III 497 E. 3.4; Lukas Heckendorn Urscheler Gedanken zur Methode der richterlichen Rechtsvergleichung im Bereich des Zivilrechts, in: Schmid/Morawa/Heckendorn Urscheler Die Rechtsvergleichung in der Rechtsprechung, Praxis, Legitimität und Methodik, 2014, S. 89 ff., S. 104 f.). Eine eigentliche Rechtsvergleichung setzt eine richtige Formulierung der Fragestellung, die Auswahl einer geeigneten Vergleichsrechtsordnung sowie die Erfassung des relevanten Stoffes voraus (Arnold F. Rusch Methoden und Ziele der Rechtsvergleichung, in: Jusletter vom 13. Februar 2006, S. 7). Vorliegend liegt der rechtsvergleichenden Untersuchung die Fragestellung der Bedeutung und Vergleichbarkeit einer Nichtverlängerungserklärung bei befristeten Arbeitsverhältnissen mit Kündigungen zugrunde. Die zu beantwortende Rechtsfrage stellt sich in gleicher Weise im deutschen wie im schweizerischen Recht. Die deutsche Rechtsordnung, insbesondere das deutsche Bühnenarbeitsrecht, erscheint aufgrund seiner massgeblichen Beeinflussung des schweizerischen Bühnenarbeitsrechtes ohnehin als besonders geeignet, um bezüglich dieser spezifischen Rechtsfrage als Auslegungselement herangezogen zu werden. Die herrschende deutsche Rechtsmeinung anerkennt, wie gesehen (siehe Ziff. 2.5.6.2.2.), die Nichtverlängerungsmitteilung als eine von einer Kündigung unterschiedliche Rechtsfigur, die nicht zur Anwendung der Kündigungsregeln führt. Namentlich gilt nicht der allgemeine Kündigungsschutz (BAG 23.10.1991 - 7 AZR 56/91 E. 4a; BOSchG 36/1993, 23/1997, www.buehnengenossenschaft.de, Schaub/Koch/Linck/Treber/ Vogelsang § 186 Rn. 8., S. 2011; Kurz/Kehrl/Nix, S. 444 Rn. 6; Opolony NZA 2001, S. 1352).

Vergleichbares gilt für das österreichische Bühnenarbeitsrecht. Auch das Theaterarbeitsgesetz (TAG) sieht in $\S 27$ Abs. 1 für den Spielzeitenvertrag folgende Regelung vor:

„Ist das Bühnenarbeitsverhältnis für bestimmte Zeit und mindestens für ein Jahr eingegangen worden, hat der/die Theaterunternehmer/in dem Mitglied bis zum 31. Jänner des Jahres, in dem das Arbeitsverhältnis endet, schriftlich mitzuteilen, dass das Arbeitsverhältnis nicht verlängert wird. Unterbleibt die Mitteilung oder erfolgt sie verspätet, gilt das Arbeitsverhältnis für ein weiteres Jahr verlängert, sofern das Mitglied dem/der Theaterunternehmer/in nicht bis spätestens zum 15. Februar des Jahres, in dem das Arbeitsverhältnis endet, schriftlich mitteilt, dass es mit einer Verlängerung des Arbeitsverhältnisses nicht einverstanden ist."

Die ständige Rechtsprechung qualifiziert die Nichtverlängerungserklärung nicht als Kündigung, sodass auch eine analoge Anwendung des § 105 ArbVG und damit eine Geltendmachung wesentlicher Arbeitnehmerschutzvorschriften nicht in Frage kommt (Michaela Weybora (Trippl) Österreichisches und schweizerisches Bühnenarbeitsrecht im Rechtsvergleich, 2014, S. 53 ff., 57, 66; Gerda Tischler Der Bühnenarbeitsvertrag und seine Beendigung, 2011, S. 39 f.; Gerda Ercher/Erwin Rath Nochmals zu den Neuerungen im Bühnenarbeitsrecht, ASoK 2011, S. 180 ff.; Katharina Urleb Das neue Arbeitsrecht in Theaterbetrieben, ASoK 2011, S. 53 ff.; Christian Kuhn Kündigung und Nichtverlängerungserklärung, ASoK 2005, S. 214 ff.; Andrea Schwarz Historische Entwicklung und aktueller Stand des Schauspielerrechts, 2005, S. 119 f.).

2.5.7. Zu prüfen ist im Folgenden, ob die Befristung des von den Parteien abgeschlossenen Spielzeitenvertrages sachlich begründet ist (siehe Ziff. 2.4.). Die Beklagte beruft sich dabei auf das Abwechslungsbedürfnis des Theaters und des Publikums, das sich sehr stark über den Spielplan bzw. die Spielzeit definiere. Dies bedinge auch bezüglich der Personalentscheidungen eine gewisse Flexibilität. Zudem sei die Theaterarbeit von den grossen künstlerischen Freiheiten des Intendanten geprägt, mit denen möglicherweise nicht jedes Bühnenmitglied künstlerisch einverstanden sei. Bei Intendantenwechseln fänden denn auch oft Änderungen im Ensemble statt. Ferner habe die Befristung der Bühnenarbeitsverträge für die Bühnenmitglieder insofern einen gewichtigen Vorteil, als sie aufgrund der Vereinheitlichung der Spielzeiten und der zulässigen Termine für die Nichtverlängerungserklärungen im europäischen Umfeld deutlich bessere Chancen hätten, auf den Anfang der nächsten Spielzeit eine neue Anstellung zu finden [...]. Nach Ansicht der Klägerin geht der Hinweis auf die Sachgerechtigkeit der (angeblichen) Befristung fehl. Sicherlich erscheine es angesichts der für einen Grossteil der Theater im deutschsprachigen Raum geltenden Spielzeiten zumindest bei Bühnenkünstlern im engeren Sinn, zu denen sie jedoch nicht zu zählen sei, angebracht, keine Beendigungsmöglichkeit eines Vertrages während einer laufenden Spielzeit vorzusehen. Dies könne jedoch ebenso gut wie durch befristete Verträge mit einer Beschränkung der Kündigungsmöglichkeiten auf lediglich einen Termin pro Jahr und Staffelung der Kündigungsfristen nach Dienstjahr erreicht werden. Dadurch werde dem Arbeitnehmer ein besserer Schutz geboten [...].

2.5.7.1. Kettenarbeitsverträge sind zulässig, wenn besondere wirtschaftliche oder soziale Umstände als sachliche Gründe den wiederholten Abschluss befristeter Verträge rechtfertigen 
(BGE 139 III 145 E. 4.1 = Pra 2013 Nr. 96). Als sachliche Gründe bei Bühnenarbeitsverträgen werden vor allem das Abwechslungsbedürfnis des Publikums und der Bühnen sowie das Eigeninteresse der Bühnenmitglieder angeführt (Mosimann Bühnenarbeitsrecht, S. 682, Rn. 127; Schellenberg S. 76 ff.; Urteil des Gewerblichen Schiedsgerichts Basel-Stadt vom 30.10.1997, Rechtsprechungsbericht 1998-1999, S. 46 E. 3b und C, www. zivilgericht.bs.ch). Was dabei zur Rechtfertigung von Kettenarbeitsverträgen ausreicht, muss umso mehr für bloss einmalige Befristungen genügen. Die Anforderungen an die sachlichen Gründe können jedenfalls nicht höher sein, weil ein wiederholter Abschluss von Bühnenarbeitsverträgen tendenziell gesteigerte Voraussetzungen an eine Rechtfertigung stellt, um eine Gesetzesumgehung auszuschliessen. In diesem Sinne stellt nach der bundesgerichtlichen Rechtsprechung denn auch das Aneinanderreihen von bloss zwei Verträgen im Allgemeinen keinen Rechtsmissbrauch dar (BGE 139 III 145 E. 4.1 = Pra 2013 Nr. 96; siehe Ziff. 2.4.), der sich Lehre und Rechtsprechung mehrheitlich angeschlossen haben (Ertl, S. 159 Rz. 559). Eine allfällige Gesetzesumgehung hat ohnehin diejenige Partei zu beweisen, die sie behauptet (BGE 139 III 145 E. 4.2.4 = Pra 2013 Nr. 96).

Rechtsvergleichend kann wiederum auf das deutsche und das österreichische Arbeitsrecht, insbesondere Bühnenarbeitsrecht, verwiesen werden. Sowohl im deutschen als auch im österreichischen Arbeitsrecht werden für die Befristung von Arbeitsverträgen, namentlich für deren Aneinanderreihung zu Kettenarbeitsverträgen, sachliche Gründe verlangt (Für das deutsche Recht: § 14 Teilzeit- und Befristungsgesetz, TzBfG; BAG 23.10.1991 - 7 AZR 56/91 E. 3 m.H.; BOSchG Hamburg 17/1995, BOSchG 23/1997, www.buehnengenossenschaft.de; Kurz/Kehrl/ Nix, S. 121 f. Rn. 16a; Nix/Hegemann/Hemke (Hrsg.) § 61 Rn. 3 ff., S. 226 f.; Urban, S. 49 ff.; Bettina Stefanie Maaß Zulässigkeit von einzel- und tarifvertraglichen Befristungsabreden im Bühnenarbeitsrecht, 2004, S. 17 ff.; Opolony ZfA 2000, S. 189 ff.; Tanja Schulz Bühnenschiedsgerichtsbarkeit und befristeter Bühnenanstellungsvertrag, 1998, S. 148 ff., 176 ff.; Riepenhausen Das Arbeitsrecht der Bühne, S. 64 ff., 195 f., insbes. Fn. 4; für das österreichische Recht: Weybora (Trippl) S. 61 ff.; Schwarz S. 120 f.).

2.5.7.2. Beim Abwechslungsbedürfnis des Publikums als sachlichen Grund für die Vertragsbefristung wird davon ausgegangen, dass die Bühnen sich nicht nur nach den verschiedenen künstlerischen Strömungen, sondern auch danach ausrichten, was der Geschmack des Publikums erwartet. Die Bühnen sind, um Erfolg zu haben, vor allem von der Gunst des Publikums abhängig. Der Geschmack des Publikums ändert sich bekanntlich laufend. Er bezieht sich nicht bloss auf die gespielten Stücke, sondern besonders auch auf die Bühnenkünstler, die an ihrer kreativen Leistung gemessen werden. In den Augen des Publikums können sich die Bühnenkünstler dabei mehr oder weniger schnell verbrauchen. Das Publikum wünscht sich dann neue Gesichter und Stimmen für die Bühne. Zum Abwechslungsbedürfnis des Publikums kommt jenes der Bühnen selber hinzu. Wie sich das Ensemble eines Theaters zusammensetzt, hängt massgeblich von der Person des Intendanten und vom fest- gelegten Spielplan ab. Der Intendant verpasst der Bühne sein künstlerisches Konzept, das auf seinem persönlichen Kunstverständnis beruht. Ihm wird allgemein die Freiheit zugestanden, das Ensemble nach seinen künstlerischen Vorstellungen zusammenzustellen und zu verändern. Zudem wirkt sich der Spielplan auf die Grösse und Zusammensetzung des Ensembles aus (BAG 23.10.1991 - 7 AZR 56/91 E. 3a; BAG 21.5.1981 - 2 AZR 1117/78 Rn. 29 ff.; Opolony ZfA 2000, S. 190 ff. m.H. auf weitere BAG-Urteile; Schulz S. 183 ff.; Tischler S. 27; Maaß S. 172 ff.; Urban S. 57 ff.).

Die Abwechslungsbedürfnisse von Publikum und Bühnen bestehen nicht bezüglich aller Bühnenkünstler im selben Ausmass. Es gibt durchaus Unterschiede unter den verschiedenen Arten von darstellenden und nicht darstellenden Bühnenkünstlern, die zu beachten sind (Tischler S. 27; siehe Ziff. 1.4.). Als Dramaturgin für Konzert und Theater bei der Beklagten zählte die Klägerin klarerweise zu den Bühnenmitgliedern (siehe Ziff. 1.4.). Hingegen war sie weder Schauspielerin, noch Sängerin, noch Tänzerin und somit nicht ein darstellendes Ensemblemitglied. Wie weit auch Dramaturgen einem Abwechslungsbedürfnis unterliegen, ist nicht unumstritten (kritisch: Götz von Olenhusen Zeitverträge, Kündigungsschutz und Tarifautonomie am Beispiel der sog. Künstlerverträge, UFITA 1976, S. 46; Tischler S. $27 \mathrm{f}$.). Richtig an den kritischen Stimmen ist, dass Dramaturgen weniger von einem Abwechslungsbedürfnis des Publikums, als vielmehr von jenem der Bühne selber betroffen sind. Die Freiheit des Intendanten zu bestimmen, mit welchem Ensemble er zusammenarbeiten kann und will, um seine künstlerischen Vorstellungen auf der Bühne umzusetzen und zu verwirklichen, bezieht sich ganz massgebend auch auf die Dramaturgie. Dramaturgen entwickeln die Spielpläne und unterstützen als Sachverständige die Intendanz und die Regie. Sie sind in die Vorbereitung und die Proben der Produktionen eingebunden und gehören damit auch zur Regie, die sie kritisch begleiten. Zudem treten sie vermittelnd als Bindeglied zwischen Intendanz, Regie und Ensemble auf. All dies verleiht ihnen eine Schlüsselstellung, die zu einem erfolgreichen Wirken und Gelingen an den Bühnen entscheidend beiträgt.

2.5.7.3. Ferner wird als sachlicher Grund für die zeitliche Befristung von Bühnenengagementsverträgen das Eigeninteresse der Bühnenkünstler angeführt. Danach streben Bühnenkünstler Engagementswechsel an oder sind innen jedenfalls nicht abgeneigt, weil sie durch die Arbeit an unterschiedlichen Bühnen im In- und Ausland sich künstlerisch weiterentwickeln und wertvolle Erfahrungen sammeln können, die ihren Bekanntheitsgrad bei anderen Bühnen, dem Publikum und in den Medien zu steigern vermögen. Engagementswechsel stehen zudem deshalb in ihrem eigenen Interesse, wenn sie ungehindert dem Regisseur oder der Intendanz an deren neuen Wirkungsort folgen wollen (BAG 23.10.1991 - 7 AZR 56/91 E. 3a; BAG 21.5.1981 - 2 AZR 1117/78 Rn. 31 f.; Schulz S. 179 ff.; Urban S. 60 f.). Auch dieser als sachlich angegebene Grund ist nicht ohne Widerspruch geblieben. Dabei wird nicht grundsätzlich in Frage gestellt, dass ein derartiges Eigeninteresse besteht. Vielmehr wird das wahre Ausmass dieses Interesses der Bühnenkünstler bezweifelt und 
davon ausgegangen, dass es durch den Abschluss unbefristeter Arbeitsverträge in gleicher oder gar besserer Weise berücksichtigt werden kann (Schulz S. 179 f.; Urban S. 61), wie auch die Klägerin argumentiert. Dabei wird übersehen, dass der Kündigungsschutz, wie er für unbefristete Arbeitsverhältnisse gilt, die Planung und Gestaltung der Zusammensetzung des Ensembles einer Bühne im Hinblick auf die Spielzeiten behindert. Der zeitliche Kündigungsschutz nach Art. 336c OR sieht vor, dass die Kündigungsfristen bei den dort genannten Gründen längstens während den festgelegten Sperrfristen stillstehen. Dies führt dazu, dass das Ende der Arbeitsverhältnisse meistens nicht mit dem Ende der Spielzeiten in Übereinstimmung steht. Dies liegt nicht im Interesse der Bühnenkünstler, weil sie für die restliche Spielzeit nach dem verlängerten Ende des Arbeitsverhältnisses kaum neue Engagements finden. Zudem widerspricht dies auch dem Abwechslungsbedürfnis der Bühnen selber, weil Bühnenkünstler, die eigentlich nicht mehr zum Ensemble gehören, weiterhin angestellt bleiben und schwerlich in den Produktionen eingesetzt werden können. Das Abwechslungsbedürfnis des Publikums trägt dabei das seine dazu bei.

Einschränkend wird geltend gemacht, dass das Veränderungsbedürfnis des Künstlers eine Befristung nur auf dessen ausdrücklichen Wunsch hin rechtfertigen kann (Rehbinder UFITA 1980, S. 98; Opolony ZfA 2000, S. 197). Diese Einschränkung übersieht, dass der Bühnenkünstler beim Abschluss des Bühnenengagementsvertrages meistens noch über kein Anschlussengagement verfügt und sich daher regelmässig darüber auch nicht ausdrücklich erklärt, sondern, wenn er entsprechende, aber noch unbestimmte Pläne für eine künftige Veränderung hat, den befristet abgeschlossenen Bühnenengagementsvertrag ohne weiteres mit der Bühne abschliesst. Daher darf gleichwohl von einem gewissen Eigeninteresse des Bühnenkünstlers ausgegangen werden. Allerdings ist auch bezüglich dieses Eigeninteresses auf die individuellen Verhältnisse abzustellen (Opolony ZfA 2000, S. 198). Die Bühnenkünstler haben ein unterschiedlich ausgeprägtes Interesse an einem Engagementswechsel. Dieses Interesse dürfte bei den darstellenden Bühnenkünstlern grundsätzlich am stärksten sein. Aber auch für nichtdarstellende Bühnenkünstler kann die Möglichkeit von Engagementswechseln im Vordergrund ihres eigenen Interesses stehen.

Dies gilt insbesondere für Dramaturgen, die durch ihre Arbeit an unterschiedlichen Bühnen ein Repertoire aufbauen und erweitern können, vor unterschiedliche Anforderungen an verschiedenartigen Bühnen gestellt sind und Konzepte entwickeln sowie Erfahrungen an ausländischen Bühnen sammeln können. Beispielhaft hierfür steht der berufliche Werdegang der Klägerin als Dramaturgin und Regieassistentin selber, die vor ihrem Engagement bei der Beklagten bereits an Bühnen und Festivals in Deutschland, den USA und Irland arbeitete [...] und sich dadurch ein gewisses Renommee erworben hatte, das es ihr zweifellos massgebend erleichterte, später wiederum in den USA und in der Schweiz engagiert zu werden [...]. Es ist daher von einem grundsätzlichen Eigeninteresse der Klägerin an Engagementswechseln auszugehen.

2.5.7.4. Vorliegend sind das Abwechslungsbedürfnis der beklagten Partei und das Eigeninteresse der Klägerin bezüglich Engagementswechseln als ausreichende sachliche Gründe für eine Befristung des auf eine Spielzeit beschränkten Bühnenengagementsvertrages festzustellen. Sie müssen genügen, umso mehr die Klägerin erstmals mit einem befristeten Vertrag von der Beklagten angestellt wurde, der nicht verlängert wurde, und es sich nicht um eine Kettenbefristung handelt. Ein solches Kettenarbeitsverhältnis liegt auch nicht unter der Hypothese der Klägerin vor, dass ihr Arbeitsverhältnis erst am 30. Juni 2017 enden würde und dannzumal drei Spielzeiten gedauert hätte, weil lediglich die Nichtverlängerung dieses ersten befristeten Vertrages in Frage steht. Nach alledem kann das vorliegende Arbeitsverhältnis nicht als unbefristetes gelten, auf das die Kündigungsschutzbestimmungen, insbesondere Art. 336c Abs. 1 lit. c OR bei Schwangerschaft und nach der Niederkunft, anzuwenden wären (vgl. AGE vom 14.7.1998, Verf. Nrn. 10001003/1997, E. 2c, S. 6: keine Anwendung von Art. 336c OR selbst bei Annahme der Nichtverlängerungserklärung als Kündigung; vgl. für das deutsche Recht: BOSchG 23/1997, www.buehnengenossenschaft.de, und BAG 23.10.1991 - 7 AZR 56/91 E. 4: keine Anwendung des während der Schwangerschaft und der anschliessenden Schutzfrist geltenden Kündigungsschutzes nach $\S 9$ Abs. 1 Mutterschutzgesetz, MuSchG auf die Nichtverlängerungsmitteilung).

2.5.8. Nach Art. 29 GAV Solo 2014 sind die zur Gültigkeit der Nichtverlängerungserklärung festgelegten Fristen und die Schriftform, in der sie vorzubereiten und durchzuführen ist, einzuhalten (siehe Ziff. 2.5.3.). Die Klägerin behauptet nicht, dass diese Anforderungen vorliegend unbeachtet blieben [...]. [...] Damit endete der Bühnenarbeitsvertrag am vereinbarten Termin, am 30. Juni 2014. Lohnansprüche darüber hinaus bestehen keine. Dies führt zur vollumfänglichen Abweisung der Klage.

2.6. Das Bühnenschiedsgericht stellt klar, dass mit dem vorliegenden Entscheid die sachlichen Gründe für eine Befristung von Bühnenengagementsverträgen, insbesondere die Rechtfertigung von Kettenarbeitsverträgen, nicht generell und pauschal bejaht werden. Vielmehr ist jede strittige Arbeitsbeziehung zwischen Theater und Bühnenkünstler für sich gesondert zu beurteilen. Dabei massgebend sind der anwendbare GAV im Bühnenbereich sowie die konkreten Umstände der zu beurteilenden Arbeitsbeziehung der Parteien, namentlich Art und Stellung des betreffenden Bühnenkünstlers und die Anzahl der sich aufeinanderfolgenden Spielzeitenverträge.

\section{$[\ldots]^{\prime \prime}$}

(Der Schiedsspruch wurde vor dem Bundesgericht nicht angefochten und ist rechtskräftig.) 